\title{
660.
}

\section{ON THE CORRESPONDENCE OF HOMOGRAPHIES AND ROTATIONS.}

[From the Mathematische Annalen, t. xv. (1879), pp. 238-240.]

IT is a fundamental notion in Prof. Klein's theory of the "Icosahedron" that homographies correspond to rotations (of a solid body about a fixed point): in such wise that, considering the homographies which correspond to two given rotations, the homography compounded of these corresponds to the rotation compounded of the two rotations.

Say the two homographies are $A+B p+C q+D p q=0, \quad A_{1}+B_{1} q+C_{1} r+D_{1} q r=0$, then, eliminating $q$, the compound homography is $A_{2}+B_{2} p+C_{2} r+D_{2} p r=0$, where

$$
A_{2}, B_{2}, C_{2}, D_{2}=B_{1} A-A_{1} C, B_{1} B-A_{1} D, D_{1} A-C_{1} C, D_{1} B-C_{1} D
$$

and the theorem is that, corresponding to these, we have rotations depending on the parameters $(\lambda, \mu, \nu),\left(\lambda_{1}, \mu_{1}, \nu_{1}\right),\left(\lambda_{2}, \mu_{2}, \nu_{2}\right)$ respectively, such that the third rotation is that compounded of the first and second rotations. The question arises to find the expression for the parameters of the homography in terms of the parameters of the corresponding rotation.

The rotation $(\lambda, \mu, \nu)$ is taken to denote a rotation through an angle 9 about an axis the inclinations of which to the axes of coordinates are $f, g, h$, the values of $\lambda, \mu, \nu$ then being $=\tan \frac{1}{2} 9 \cos f, \tan \frac{1}{2} 9 \cos g, \tan \frac{1}{2} 9 \cos h$ respectively: $\left(\lambda_{1}, \mu_{1}, \nu_{1}\right)$ and $\left(\lambda_{2}, \mu_{2}, \nu_{2}\right)$ have of course the like significations; and then, if $(\lambda, \mu, \nu)$ refer to the first rotation, and $\left(\lambda_{1}, \mu_{1}, \nu_{1}\right)$ to the second rotation, the values of $\left(\lambda_{2}, \mu_{2}, \nu_{2}\right)$ for the rotation compounded of these are taken to be*:

$$
\begin{aligned}
& \lambda_{2}=\lambda+\lambda_{1}+\mu \nu_{1}-\mu_{1} \nu, \\
& \mu_{2}=\mu+\mu_{1}+\nu \lambda_{1}-\nu_{1} \lambda, \\
& \nu_{2}=\nu+\nu_{1}+\lambda \mu_{1}-\lambda_{1} \mu,
\end{aligned}
$$

* The numerators might equally well have been $\lambda+\lambda_{1}-\left(\mu \nu_{1}-\mu_{1} \nu\right)$, etc., but there is no essential difference: we pass from one set of formulæ to the other by reversing the signs of all the symbols: and hence, by properly fixing the sense of the rotations, the signs may be made to be + as in the text. Assuming this to be so, if we then reverse the order of the component rotations, we have for the new compound rotation the like formulæ with the signs - instead of +; but this in passing. The formulæ, virtually due to Rodrigues, are given in my paper "On the motion of rotation of a solid body," Camb. Math. Journal, t. III. (1843), [6].

C. $\mathrm{X}$. 
each divided by

$$
1-\lambda \lambda_{1}-\mu \mu_{1}-\nu \nu_{1}
$$

and if we then write for $\lambda, \mu, \nu$, the quotients $x, y, z$ each divided by $w$, and in like manner for $\lambda_{1}, \mu_{1}, \nu_{1}$ and $\lambda_{2}, \mu_{2}, \nu_{2}$, the quotients $x_{1}, y_{1}, z_{1}$ each divided by $w_{1}$, and $x_{2}, y_{2}, z_{2}$ each divided by $w_{2}$, the formulæ for the composition of the rotations are

$$
\begin{aligned}
& x_{2}=x w_{1}+x_{1} w+y z_{1}-y_{1} z \\
& y_{2}=y w_{1}+y_{1} w+z x_{1}-z_{1} x \\
& z_{2}=z w_{1}+z_{1} w+x y_{1}-x_{1} y, \\
& w_{2}=w w_{1}-x x_{1}-y y_{1}-z z_{1}
\end{aligned}
$$

and the question is to express $A, B, C, D$ as functions of $(x, y, z, w)$, such that $A_{1}, B_{1}, C_{1}, D_{1}$ denoting the like functions of $\left(x_{1}, y_{1}, z_{1}, w_{1}\right), A_{2}, B_{2}, C_{2}, D_{2}$ shall denote the like functions of $\left(x_{2}, y_{2}, z_{2}, w_{2}\right)$.

It is found that the required conditions are satisfied by assuming

$$
A, B, C, D=i x-y,-i z+w,-i z-w,-i x-y,
$$

(where $i=\sqrt{-1}$ as usual): in fact, we then have

$$
\begin{aligned}
A_{2} & =B_{1} A-A_{1} C \\
& =\left(-i z_{1}+w_{1}\right)(i x-y)-\left(i x_{1}-y_{1}\right)(-i z-w) \\
& =i\left(x w_{1}+x_{1} w+y z_{1}-y_{1} z\right)-\left(y w_{1}+y_{1} w+z x_{1}-z_{1} x\right) \\
& =-y_{2}+i x_{2},
\end{aligned}
$$

as it should be: and we verify in like manner the values of $B_{2}, C_{2}$ and $D_{2}$ respectively.

The result consequently is that we have the homography

$$
(i x-y)+(-i z+w) p+(-i z-w) q+(-i x-y) p q=0
$$

corresponding to the rotation $\left(\frac{x}{w}, \frac{y}{w}, \frac{z}{w}\right)$ : where $\frac{x}{w}, \frac{y}{w}, \frac{z}{w}$ are the parameters of rotation, $\tan \frac{1}{2} 9 \cos f, \tan \frac{1}{2} 9 \cos g, \tan \frac{1}{2} 9 \cos h$.

I remark as regards the geometrical theory that, if we consider two lines $J$ and $K$ fixed in space, and a third line $L$ fixed in the solid body and moveable with it; then, for any given position of the solid body, the three lines $J, K, L$ are directrices of a hyperboloid, the generatrices whereof meet each of the three lines: and these generatrices determine, say on the fixed lines $J$ and $K$, two series of points corresponding homographically to each other: that is, corresponding to any given position of the solid body we have a homography. But it is not immediately obvious how we can thence obtain the foregoing analytical formulæ.

Cambridge, 3 April, 1879. 